\title{
A SHARP INEQUALITY FOR MARTINGALE TRANSFORMS AND THE UNCONDITIONAL BASIS CONSTANT OF A MONOTONE BASIS IN $L^{p}(0,1)$
}

\author{
K. P. CHOI
}

Abstract. Let $1<p<\infty$. Let $d=\left(d_{1}, d_{2}, \ldots\right)$ be a real-valued martingale difference sequence, $\theta=\left(\theta_{1}, \theta_{2}, \ldots\right)$ is a predictable sequence taking values in $[0,1]$. We show that the best constant of the inequality,

satisfies

$$
\left\|\sum_{k=1}^{n} \theta_{k} d_{k}\right\|_{p} \leq c_{p}\left\|\sum_{k=1}^{n} d_{k}\right\|_{p}, \quad n \geq 1
$$

$$
c_{p}=\frac{p}{2}+\frac{1}{2} \log \left(\frac{1+\gamma}{2}\right)+\frac{\alpha_{2}}{p}+\cdots,
$$

where $\gamma=e^{-2}$ and $\alpha_{2}=\left[\frac{1}{2} \log \frac{1+\gamma}{2}\right]^{2}+\frac{1}{2} \log \frac{1+\gamma}{2}-2\left(\frac{\gamma}{1+\gamma}\right)^{2}$. The best constant equals the unconditional basis constant of a monotone basis of $L^{p}(0,1)$.

\section{INTRODUCTION}

More than fifty years ago, Paley [10] proved the following inequality for the Walsh system of functions $\psi_{n}$ on the Lebesgue unit interval. If $1<p<\infty$, there is a positive real number $c_{p}$ with the property that if $b_{1}, b_{2}, \ldots$ are real numbers and

$$
e_{n}=\sum_{2^{n} \leq m<2^{n+1}} b_{m} \psi_{m}
$$

then

$$
c_{p}^{-1}\left\|\sum_{k=1}^{n} e_{k}\right\|_{p} \leq\left\|\sum_{k=1}^{n} \varepsilon_{k} e_{k}\right\|_{p} \leq c_{p}\left\|\sum_{k=1}^{n} e_{k}\right\|_{p}
$$

for all signs $\varepsilon_{k} \in\{1,-1\}$ and all positive integer $n$. Notice that the left-hand side of this inequality follows at once from the right-hand side.

It was then observed by Marcinkiewicz [8] that Paley's inequality can be given an equivalent formulation in terms of the Haar system of functions $h_{n}$ :

$$
c_{p}^{-1}\left\|\sum_{k=1}^{n} a_{k} h_{k}\right\|_{p} \leq\left\|\sum_{k=1}^{n} \varepsilon_{k} a_{k} h_{k}\right\|_{p} \leq c_{p}\left\|\sum_{k=1}^{n} a_{k} h_{k}\right\|_{p}, \quad 1<p<\infty .
$$

Received by the editors February 9, 1989 and, in revised form, December 4, 1989.

1980 Mathematics Subject Classification (1985 Revision). Primary 60G42, 60G46; Secondary $60 \mathrm{H} 05$.

Key words and phrases. Martingale, martingale transform, zigzag martingale, stochastic integral, unconditional basis constant, Haar system, monotone basis, contractive projection, biconcave functions. 
Here $a_{1}, a_{2}, \ldots$ are real numbers and the constant $c_{p}$ is the same as in Paley's inequality.

Throughout this work, we adopt the following convention. The constant $c_{p}$ may change from one use to the next; however, if it is necessary to be more specific, the best constant in an inequality, say (1.1), is denoted by $c_{p}(1.1)$. With this notation, Marcinkiewicz's result can be stated as follows:

$$
c_{p}(1.1)=c_{p}(1.2) .
$$

In 1966, Burkholder [1] extended the result of Paley and Marcinkiewicz to martingales:

$$
\left\|\sum_{k=1}^{n} v_{k} d_{k}\right\|_{p} \leq c_{p}\left\|\sum_{k=1}^{n} d_{k}\right\|_{p}, \quad 1<p<\infty,
$$

where $v=\left(v_{1}, v_{2}, \ldots\right)$ is a predictable sequence uniformly bounded in absolute value by 1 and $d=\left(d_{1}, d_{2}, \ldots\right)$ is a martingale difference sequence. Here the constant $c_{p}$ is independent of both $v$ and $d$.

An important special case of $(1.3)$ is

$$
\left\|\sum_{k=1}^{n} \varepsilon_{k} d_{k}\right\|_{p} \leq c_{p}\left\|\sum_{k=1}^{n} d_{k}\right\|_{p}, \quad 1<p<\infty,
$$

where, again, $\varepsilon_{k} \in\{1,-1\}$. Clearly, $c_{p}(1.4) \leq c_{p}(1.3)$. In 1981, Burkholder [2] showed that $c_{p}(1.3) \leq c_{p}(1.4)$, so equality holds. The Haar system $h=$ $\left(h_{1}, h_{2}, \ldots\right)$ is a martingale difference sequence, as is $d=\left(a_{1} h_{1}, a_{2} h_{2}, a_{3} h_{3}, \ldots\right)$ for real numbers $a_{k}$. Therefore $c_{p}(1.2) \leq c_{p}(1.4)$. Maurey [9] proved the reverse inequality. Therefore

$$
c_{p}(1.1)=c_{p}(1.2)=c_{p}(1.3)=c_{p}(1.4) .
$$

In 1984, Burkholder [3] derived the value of this best constant. It is

$$
p^{*}-1
$$

where $p^{*}$ is the maximum of $p$ and its conjugate $q=p /(p-1)$. The proof rests on solving a system of nonlinear partial differential equations and inequalities. See his paper [4] for a shorter proof.

Inequality (1.3) carries over to stochastic integrals with no change in the value of the best constant (see [3]). It has applications not only in probability theory but also in Fourier analysis and the theory of singular integrals. It carries over to $B$-valued martingales for a large class of Banach spaces $B$ where the constant depends both on $p$ and $B$. A geometrical characterization of this class is given in [2]. For a discussion of some of this, see [5].

The main contribution of this paper is a set of equations (see Theorem 3.3 and (3.11), (3.12) in §3) that determine the best constant in the inequality,

$$
\left\|\sum_{k=1}^{n} \theta_{k} d_{k}\right\|_{p} \leq c_{p}\left\|\sum_{k=1}^{n} d_{k}\right\|_{p} .
$$

Here, as before, $1<p<\infty$ and $d=\left(d_{1}, d_{2}, \ldots\right)$ is a martingale difference sequence, but $\theta=\left(\theta_{1}, \theta_{2}, \ldots\right)$ is a predictable sequence taking values 0 or 1 . 
The gambling interpretation of (1.5) is obvious: as long as the gambler cannot look into the future and the game is fair in the sense that $d=\left(d_{1}, d_{2}, \ldots\right)$ forms a martingale difference sequence then his fortune $\sum_{k=1}^{n} \theta_{k} d_{k}$ is controlled by the fortune $\sum_{k=1}^{n} d_{k}$ that would have been achieved without skipping bets. This holds for any $p$ in $(1, \infty)$, but does not hold for $p=1$ or $p=\infty$ in general. Inequality $(1.5)$ can be extended to $\theta_{k}$ taking values in $[0,1]$ with the same optimal constant $c_{p}(1.5)$.

Using a discretization argument (see $\S 16$ of [3]), we can extend the inequality (1.5) to stochastic integrals with $c_{p}(1.5)$ as the best constant.

The inequality (1.5) has another important connection, a connection with the unconditional basis constant. Let $1<p<\infty$ and $e=\left(e_{1}, e_{2}, \ldots\right)$ be a basis of real $L^{p}(0,1)$. The unconditional basis constant, denoted by $K_{p}(e)$, is the extended real number (see [7], for example)

$$
\begin{array}{r}
\sup \left\{\left\|\sum_{k=1}^{n} \theta_{k} a_{k} e_{k}\right\|_{p}: \text { where }\left\|\sum_{k=1}^{n} a_{k} e_{k}\right\|_{p}=1, \text { for } n \geq 1,\right. \\
\left.a_{1}, \ldots, a_{n} \text { are real numbers and } \theta_{k} \in\{0,1\}\right\} .
\end{array}
$$

Clearly, the unconditional basis constant of the Haar system satisfies

$$
K_{p}(h) \leq c_{p}(1.5)
$$

and by the method of Maurey [9] the reverse inequality is true. Therefore, we have the following theorem.

Theorem A. If $K_{p}(h)$ is the unconditional basis constant of the Haar system, then

$$
K_{p}(h)=c_{p}(1.5), \quad 1<p<\infty .
$$

Let $(\Omega, \mathscr{A}, \mu)$ be a positive measure space and $\left(P_{1}, P_{2}, \ldots\right)$ be a nondecreasing sequence of contractive projections in $L^{p}(\Omega, \mathscr{A}, \mu)$ : for every $n, m \geq 1$,

$$
P_{m} P_{n}=P_{n} P_{m}=P_{\min \{m, n\}} \text { and }\left\|P_{n}\right\| \leq 1 .
$$

Theorem B. Let $P=\left(P_{1}, P_{2}, \ldots\right)$ be any nondecreasing sequence of contractive projections in $L^{p}(\Omega, \mathscr{A}, \mu)$ and let $P_{0}=0$. If $f \in L^{p}(\Omega, \mathscr{A}, \mu)$, then

$$
\left\|\sum_{k=1}^{n} a_{k}\left(P_{k}-P_{k-1}\right) f\right\|_{p} \leq c_{p}(1.5)\|f\|_{p}, \quad 1<p<\infty,
$$

for all integers $n \geq 1$ and all numbers $a_{k} \in[0,1]$. And this inequality is sharp.

A basis (for definition, see [7]) $e=\left(e_{1}, e_{2}, \ldots\right)$ in a real Banach space $B$ with norm $\|\cdot\|_{B}$ is said to be monotone if

$$
\left\|\sum_{k=1}^{n} a_{k} e_{k}\right\|_{B} \leq\left\|\sum_{k=1}^{n+1} a_{k} e_{k}\right\|_{B}
$$

for every $n \geq 1$ and all $a_{k} \in \mathbf{R}$. If $B$ does have a monotone basis and $x=\sum_{k=1}^{\infty} a_{k} e_{k}$, then let $P_{0} x=0$ and $P_{n} x=\sum_{k=1}^{n} a_{k} e_{k}$ for all $n \geq 1$. Then it is easy to verify that $P=\left(P_{0}, P_{1}, \ldots\right)$ is a nondecreasing sequence of contractive projections so we have the following conclusion. 
Corollary $\mathbf{C}$. The unconditional basis constant of a monotone basis of $L^{p}(0,1)$ is $c_{p}(1.5)$, i.e.

$$
K_{p}(e)=c_{p}(1.5) .
$$

See [3] for the proof of Theorem B. These theorems illustrate the interest in knowing the value of $c_{p}(1.5)$.

In $\S 4$ (see Theorem 4.3), it is shown that

$$
K_{p}(e)=c_{p}(1.5) \sim \frac{p}{2}+\frac{1}{2} \log \left(\frac{1+e^{-2}}{2}\right) .
$$

\section{ZigZAG MARTINGALES}

Let $f=\left(f_{1}, f_{2}, \ldots\right)$ be a real-valued martingale on a probability space $(\Omega, \mathscr{A}, P)$ and $d=\left(d_{1}, d_{2}, \ldots\right)$ be its difference sequence. Suppose $f_{1}=$ $x-y$, where $x, y \in \mathbf{R}$ and $\theta=\left(1, \theta_{2}, \theta_{3}, \ldots\right)$ is a sequence of real numbers taking values in $\{0,1\}$. A sequence $g=\left(g_{1}, g_{2}, \ldots\right)$ is the transform of $f$ by $\theta=\left(1, \theta_{2}, \theta_{3}, \ldots\right)$ if, for every $n \geq 1, g_{n}=\sum_{k=1}^{n} \theta_{k} d_{k}$, where $\theta_{1}=1$. Indeed, $g$ is also a real-valued martingale.

Let $\left(X_{1}, Y_{1}\right)=(x, y)$ and for all $n \geq 2$,

$$
\begin{aligned}
& X_{n}=x+\sum_{k=2}^{n} \theta_{k} d_{k}, \\
& Y_{n}=y+\sum_{k=2}^{n}\left(\theta_{k}-1\right) d_{k} .
\end{aligned}
$$

Then $Z=\left(Z_{1}, Z_{2}, \ldots\right)$, where $Z_{n}=\left(X_{n}, Y_{n}\right)$, is an $\mathbf{R}^{2}$-valued martingale starting at $(x, y)$. Since $\theta_{k} \in\{0,1\}$, it is obvious that for each $n \geq 2$ either $X_{n}-X_{n-1} \equiv 0$ or $Y_{n}-Y_{n-1} \equiv 0$. In other words, if $Z$ moves at all at the $n$th step $(n \geq 2)$, it moves either horizontally or vertically, which way depending on $n$ only. In the terminology of [3], $Z$ is a zigzag martingale. Furthermore, we can recover $f_{n}$ and $g_{n}$ by

$$
\begin{gathered}
f_{n}=X_{n}-Y_{n}, \\
g_{n}+y=X_{n} .
\end{gathered}
$$

\section{SHARP INEQUALITIES}

By a standard duality argument, it can be proved that

$$
c_{p}(1.5)=c_{q}(1.5), \quad 1<p, q<\infty, \frac{1}{p}+\frac{1}{q}=1 .
$$

Therefore, we will determine $c_{p}(1.5), 2<p<\infty$.

Let $p_{0}$ be the unique solution to the equation

$$
\begin{gathered}
p-2=\left[\frac{(p-1)(p-2)}{-p^{2}+5 p-5}\right]^{p-1}, \quad 2<p<3, \\
p_{0} \simeq 2.5455458 .
\end{gathered}
$$


Indeed, putting $x=p-2$ into (3.2) and simplifying, we obtain an equivalent equation

(3.4) $(x+1) \log \left(-x^{2}+x+1\right)=x \log x+(x+1) \log (x+1), \quad 0<x<1$.

The left-hand side is concave in $x$ and the right-hand side is convex in $x$. The existence and uniqueness of solution to (3.4) can then be deduced readily by considering the behavior of the two sides near the endpoints of $(0,1)$. Indeed, we have

$$
(p-2)\left[-p^{2}+5 p-5\right]^{p-1} \geq[(p-1)(p-2)]^{p-1}, \quad 2<p \leq p_{0},
$$

and,

$$
(p-2)\left[-p^{2}+5 p-5\right]^{p-1}<[(p-1)(p-2)]^{p-1}, \quad p_{0}<p \leq 3 .
$$

For $t \in[-1,1]$, we define

$$
\begin{aligned}
& E(t)=\operatorname{sgn}(t)|t|^{p-1}-(p-1) t+p-2, \\
& A(t)=(p-1)(1-t)^{2}-[(p-2)-p t] E(t), \\
& D(t)=(p-1)(1-t)^{2}+t E(t), \quad \text { and } \\
& B(t)=(p-1)(1-t)^{2} E(t)-t A(t),
\end{aligned}
$$

or equivalently,

$$
B(t)=[(p-1)-p t] E(t)-t D(t) .
$$

For $p_{0}<p<\infty$, let $I_{p}=(0 \vee(p-3) /(p-1),(p-2) / p)$, and for $2<p \leq$ $p_{0}, I_{p}=(-(3-p) / 2 p, 0]$.

Lemma 3.1. For $2<p<\infty$, there exists a unique solution, $t_{p} \in I_{p}$, to the equation

$$
[p-2-(p-1) t][A(t)]^{p-1}=[B(t)]^{p-1} .
$$

For $2<p<\infty$, let

$$
\begin{gathered}
k_{p}=\frac{A\left(t_{p}\right)}{(p-1)\left(1-t_{p}\right)^{2}}\left\{\frac{D\left(t_{p}\right)}{A\left(t_{p}\right)}\right\}^{p}, \quad \text { and } \\
v(x, y)=|x|^{p}-k_{p}|x-y|^{p}, \quad(x, y) \in \mathbf{R}^{2} .
\end{gathered}
$$

Lemma 3.2. For $2<p<\infty$, there exists a biconcave function $u: \mathbf{R}^{2} \rightarrow \mathbf{R}$ such that $u(0,0)=0, u(x, y) \geq v(x, y)$ for all $(x, y) \in \mathbf{R}^{2}$, and $u$ satisfies the following bounds:

$$
\begin{gathered}
|u(x, y)| \leq c_{p}\left(|x|^{p}+|y|^{p}\right), \\
\left|u_{x}(x, y)\right| \leq c_{p}\left(|x|^{p-1}+|y|^{p-1}\right), \quad \text { and }, \\
\left|u_{y}(x, y)\right| \leq c_{p}\left(|x|^{p-1}+|y|^{p-1}\right) .
\end{gathered}
$$

Lemmas 3.1 and 3.2 will be proved in $\S 5$.

The following theorem is the main result of this paper. 
Theorem 3.3. For $2<p<\infty$,

$$
c_{p}(1.5)=\left(k_{p}\right)^{1 / p}
$$

Proof. The proof is based on the idea of Burkholder in [4]. The proof consists of two parts. Part I makes use of Lemma 3.2 to show that $c_{p}^{p}(1.5) \leq k_{p}$ and Part II shows by an example that $k_{p} \leq c_{p}^{p}(1.5)$.

Part I. Let $d=\left(d_{1}, d_{2}, \ldots\right)$ be a martingale difference sequence of an $L^{p}$ bounded martingale $f$ and let $g$ be the martingale transform of $f$ by a predictable sequence $\theta=\left(\theta_{1}, \theta_{2}, \ldots\right)$, where $\theta_{k} \in[0,1]$. By a reduction argument (see $\S 2$ in [3]), we may assume $d_{1} \equiv 0, \theta_{1} \equiv 1$ and that $\theta=\left(1, \theta_{2}, \ldots\right)$ is a sequence of real numbers taking values in $\{0,1\}$. Construct the zigzag martingale $Z=\left(Z_{1}, Z_{2}, \ldots\right), Z_{n}=\left(X_{n}, Y_{n}\right)$ by $(2.1)$ and (2.2) where $x=y=0$. By Lemma 3.2, there exists a biconcave function $u(x, y)$ that majorizes $v(x, y)$, therefore

$$
v\left(X_{n}, Y_{n}\right)=\left|X_{n}\right|^{p}-k_{p}\left|X_{n}-Y_{n}\right|^{p} \leq u\left(X_{n}, Y_{n}\right) \text {. }
$$

By (2.3) and (2.4), this implies that

$$
\left|g_{n}\right|^{p}-k_{p}\left|f_{n}\right|^{p} \leq u\left(X_{n}, Y_{n}\right) .
$$

Using the bound (3.14), we see that $u\left(X_{n}, Y_{n}\right)$ is integrable. Taking expectation, we have that

$$
\left\|g_{n}\right\|_{p}^{p}-k_{p}\left\|f_{n}\right\|_{p}^{p} \leq E u\left(X_{n}, Y_{n}\right)
$$

We also observe that

$$
\begin{aligned}
u\left(X_{n}, Y_{n}\right) \leq & u\left(X_{n-1}, Y_{n-1}\right)+\theta_{n} u_{x}\left(X_{n-1}, Y_{n-1}\right) d_{n} \\
& +\left(\theta_{n}-1\right) u_{y}\left(X_{n-1}, Y_{n-1}\right) d_{n}
\end{aligned}
$$

since $u$ is biconcave and $\theta_{n}=0$ or $1-\theta_{n}=0$.

Now $d_{n}$ is $L^{p}$-integrable and by the bounds (3.15) and (3.16), it follows that $u_{x}\left(X_{n-1}, Y_{n-1}\right)$ and $u_{y}\left(X_{n-1}, Y_{n-1}\right)$ are $L^{q}$-integrable. Indeed

$$
\begin{aligned}
\left\|u_{x}\left(X_{n-1}, Y_{n-1}\right)\right\|_{q}^{q} & \leq c_{p} E\left(\left|X_{n-1}\right|^{p-1}+\left|Y_{n-1}\right|^{p-1}\right)^{q} \\
& \leq c_{p}\left(E\left|X_{n-1}\right|^{(p-1) q}+E\left|Y_{n-1}\right|^{(p-1) q}\right) \\
& =c_{p}\left(\left\|X_{n-1}\right\|_{p}^{p}+\left\|Y_{n-1}\right\|_{p}^{p}\right) \\
& =c_{p}\left(\left\|\sum_{k=1}^{n} \theta_{k} d_{k}\right\|_{p}^{p}+\left\|\sum_{k=1}^{n}\left(1-\theta_{k}\right) d_{k}\right\|_{p}^{p}\right) \\
& \leq c_{p} \sum_{k=1}^{n}\left\|d_{k}\right\|_{p}^{p}<\infty .
\end{aligned}
$$

Similarly $\left\|u_{y}\left(X_{n-1}, Y_{n-1}\right)\right\|_{q}<\infty$. By Hölder's inequality, $u_{x}\left(X_{n-1}, Y_{n-1}\right) d_{n}$ and $u_{y}\left(X_{n-1}, Y_{n-1}\right) d_{n}$ are integrable. Therefore

$$
E u_{x}\left(X_{n-1} Y_{n-1}\right) d_{n}=E\left(E\left(d_{n} \mid \mathscr{A}_{n-1}\right) u_{x}\left(X_{n-1}, Y_{n-1}\right)\right)=0
$$

and, similarly, $E u_{y}\left(X_{n-1}, Y_{n-1}\right) d_{n}=0$.

Hence, by (3.18) we get $E u\left(X_{n}, Y_{n}\right) \leq E u\left(X_{n-1}, Y_{n-1}\right)$. Working backward, we have

$$
E u\left(X_{n}, Y_{n}\right) \leq E u\left(X_{1}, Y_{1}\right)=u(0,0)=0 .
$$


Combining this and (3.17), we have

$$
\left\|g_{n}\right\|_{p}^{p} \leq k_{p}\left\|f_{n}\right\|_{p}^{p}
$$

Therefore $c_{p}^{p}(1.5) \leq k_{p}$.

Part II. We will exhibit an example here to show that $c_{p}^{p}(1.5) \geq k_{p}$. We need the following definitions. Let $t_{p}$ be as in Lemma 3.2,

$$
\begin{aligned}
\alpha & =\left[p-2-(p-1) t_{p}\right] \frac{E\left(t_{p}\right)}{D\left(t_{p}\right)}, \\
\beta & =\frac{t_{p}}{\left[p-1-p t_{p}\right]} \frac{D\left(t_{p}\right)}{E\left(t_{p}\right)}, \\
\lambda & =\left[p-1-p t_{p}\right] \frac{E\left(t_{p}\right)}{D\left(t_{p}\right)}, \\
\omega & =2 \frac{\left[p-1-p t_{p}\right]}{(p-1)\left(1-t_{p}\right)^{2}} E\left(t_{p}\right) .
\end{aligned}
$$

Note that $\beta>0$ when $p_{0}<p<\infty ; \beta \leq 0$ when $2<p \leq p_{0}$.

We need the following technical lemma which can be verified by straightforward computation. Its proof is given in $\S 5$.

Lemma 3.4. For $2<p<\infty$,

(i) $\frac{\lambda}{\lambda-\alpha}+\frac{|\beta \lambda|^{p}}{1-\beta \lambda}=k_{p}\left[\frac{(1-\alpha)^{p} \lambda}{\lambda-\alpha}+\frac{(1-\beta)^{p} \lambda^{p}}{1-\beta \lambda}\right]$,

(ii) $\frac{\lambda}{\lambda-\alpha}+\frac{1}{1-\beta \lambda}=p$,

(iii) $0<\alpha<\lambda<\beta^{-1}$, for $p_{0}<p<\infty$, and

(iii') $0<\alpha<\lambda, \beta \leq 0$, for $2<p \leq p_{0}$.

Returning to the proof of Theorem 3.3, we proceed as follows. For $x>0$, there exists a unique $\eta \in(\alpha, \lambda)$ such that

$$
x^{p}=\frac{\lambda}{\lambda-\eta}+\frac{1}{1-\beta \lambda}-p .
$$

We have, by (ii) in Lemma 3.4, that $\eta$ converges to $\alpha$ as $x$ converges to 0 . Case (1). $p_{0}<p<\infty$. Fix $x>0$ and choose $\delta \in(0, x)$, actually we will eventually let $\delta \rightarrow 0$. For all $k \geq 1$, define

$$
\begin{aligned}
& \xi_{k}=1-\frac{\lambda \delta}{(\lambda-\eta)(x+k \delta)+\eta \delta}, \\
& \gamma_{k}=1-\frac{\delta}{(1-\beta \lambda)(x+k \delta)}, \\
& \pi_{0}=1, \quad \text { and } \quad \pi_{k}=\prod_{j=1}^{n} \gamma_{j} \xi_{j} .
\end{aligned}
$$

Note that $\beta>0$.

When there is no risk of ambiguity, we use $[a, b)$ to denote either the interval $\{x: a \leq x<b\}$ or the indicator function of the set $[a, b)$.

On the probability space $([0,1), \mathscr{A}, m)$, where $\mathscr{A}$ is the $\sigma$-field of all Borel measurable sets in $[0,1)$ and $m$ is the Lebesgue measure on $[0,1)$, we 
define a sequence of functions on $[0,1), d=\left(d_{1}, d_{2}, \ldots\right)$ as follows:

$$
\begin{gathered}
d_{1} \equiv(1-\lambda) x, \quad \text { and for all } k \geq 1, \\
d_{2 k}=-\lambda \delta\left[0, \pi_{k-1} \xi_{k}\right)+(\lambda-\eta)[x+(k-1) \delta]\left[\pi_{k-1} \xi_{k}, \pi_{k-1}\right), \\
d_{2 k+1}=\delta\left[0, \pi_{k}\right)-[(1-\beta \lambda)(x+k \delta)-\delta]\left[\pi_{k}, \pi_{k-1} \xi_{k}\right)
\end{gathered}
$$

It is not difficult to see that $d=\left(d_{1}, d_{2}, \ldots\right)$ forms a martingale difference sequence. Let $\theta_{2 k}=0$ and $\theta_{2 k-1}=1$ for all $k \geq 1$. Let $f$ be the martingale with the martingale difference sequence $d=\left(d_{1}, d_{2}, \ldots\right)$ and $g$ the martingale transform of $f$ by $\theta=\left(\theta_{1}, \theta_{2}, \ldots\right)$. Therefore, we have

$$
\begin{aligned}
\|f\|_{p}= & \lim _{n \rightarrow \infty}\left\|f_{2 n+1}\right\|_{p}^{p} \\
= & \lim _{n \rightarrow \infty}\left\|X_{2 n+1}-Y_{2 n+1}\right\|_{p}^{p} \\
= & \lim _{n \rightarrow \infty}\left\{(1-\lambda)^{p}(x+n \delta)^{p} \pi_{n}+\sum_{k=1}^{n}(1-\eta)^{p}[x+(k-1) \delta]^{p} \pi_{k-1}\left(1-\xi_{k}\right)\right. \\
& \left.+\sum_{k=1}^{n}(1-\beta)^{p} \lambda^{p}(x+k \delta)^{p} \pi_{k-1} \xi_{k}\left(1-\gamma_{k}\right)\right\},
\end{aligned}
$$

and similarly,

$$
\begin{aligned}
\|g+\lambda x\|_{p}^{p}= & \lim _{n \rightarrow \infty}\left\|X_{2 n+1}\right\|_{p}^{p} \\
& =\lim _{n \rightarrow \infty}\left\{(x+n \delta)^{p} \pi_{n}+\sum_{k=1}^{n}[x+(k-1) \delta]^{p} \pi_{k-1}\left(1-\xi_{k}\right)\right. \\
& \left.\quad+\sum_{k=1}^{n}(\beta \lambda)^{p}(x+k \delta)^{p} \pi_{k-1} \xi_{k}\left(1-\gamma_{k}\right)\right\} .
\end{aligned}
$$

We make use of the inequality $1-w \delta \leq(1-\delta)^{w}$ for all $w>1$ and $0<\delta<1$. Since $\beta \lambda<1$ by Lemma 3.4(iii), we introduce $s_{1}=\lambda /(\lambda-\eta)>1$ and $s_{2}=1 /(1-\beta \lambda)>1$, then

$$
\begin{aligned}
\pi_{k} & =\left(\prod_{j=1}^{k} \gamma_{j}\right)\left(\prod_{j=1}^{k} \xi_{j}\right) \leq \prod_{j=1}^{k}\left(1-\frac{\delta}{x+j \delta}\right)^{s_{2}} \times \prod_{j=1}^{k}\left(1-\frac{\delta}{x+j \delta+\frac{\eta \delta}{\lambda-\eta}}\right)^{s_{1}} \\
& =\left(\frac{x}{x+k \delta}\right)^{s_{1}+s_{2}}\left(1+\frac{\eta \delta}{(\lambda-\eta) x}\right)^{s_{1}}\left(\frac{x+k \delta}{x+k \delta+\frac{\eta \delta}{\lambda-\eta}}\right)^{s_{1}} \\
& \leq H_{1}(\delta)\left(\frac{x}{x+k \delta}\right)^{s_{1}+s_{2}}
\end{aligned}
$$

where

$$
H_{1}(\delta)=\left(1+\frac{\eta \delta}{(\lambda-\eta) x}\right)^{s_{1}} \rightarrow 1 \quad \text { as } \delta \rightarrow 0
$$

Now

$$
0 \leq(x+n \delta)^{p} \pi_{n} \leq H_{1}(\delta)\left(\frac{x}{x+n \delta}\right)^{s_{1}+s_{2}}(x+n \delta)^{p}
$$


the right side goes to zero as $n$ goes to infinity by (3.23). So $\underset{\delta \rightarrow 0}{\limsup }\|f\|_{p}^{p}$

$$
\begin{aligned}
& \leq \limsup _{\delta \rightarrow 0}\left\{\sum_{k=1}^{\infty}(1-\eta)^{p}[x+(k-1) \delta]^{p} \pi_{k-1}\left(1-\xi_{k}\right)\right. \\
& \left.\qquad \sum_{k=1}^{\infty}(1-\beta)^{p} \lambda^{p}(x+k \delta)^{p} \pi_{k-1} \xi_{k}\left(1-\gamma_{k}\right)\right\} \\
& =\limsup _{\delta \rightarrow 0}\left\{(1-\eta)^{p} x^{p}\left(1-\xi_{1}\right)+\sum_{k=1}^{\infty}(1-\eta)^{p}(x+k \delta)^{p} \pi_{k}\left(1-\xi_{k+1}\right)\right. \\
& \left.\leq \limsup _{\delta \rightarrow 0}\left\{\frac{\lambda(1-\eta)^{p}}{\lambda-\eta}+\frac{(1-\beta)^{p} \lambda^{p}}{1-\beta \lambda}\right\} \delta \sum_{k=1}^{\infty}(x+k \delta)^{p-1} \pi_{k}(1-\beta)^{p} \lambda^{p}(x+k \delta)^{p} \pi_{k} \frac{1-\gamma_{k}}{\gamma_{k}}\right\} \\
& \leq\left\{\frac{\lambda(1-\eta)^{p}}{\lambda-\eta}+\frac{(1-\beta)^{p} \lambda^{p}}{1-\beta \lambda}\right\} \limsup _{\delta \rightarrow 0}^{\infty} H_{1}(\delta) \delta \sum_{k=1}^{\infty} \frac{x^{s_{1}+s_{2}}}{(x+k \delta)^{s_{1}+s_{2}-p+1}} \\
& \leq\left\{\frac{\lambda(1-\eta)^{p}}{\lambda-\eta}+\frac{(1-\beta)^{p} \lambda^{p}}{1-\beta \lambda}\right\} \limsup _{\delta \rightarrow 0} \int_{0}^{\infty} \frac{x^{s_{1}+s_{2}}}{(x+t \delta)^{s_{1}+s_{2}-p+1}} d t \\
& =\left\{\frac{\lambda(1-\eta)^{p}}{\lambda-\eta}+\frac{(1-\beta)^{p} \lambda^{p}}{1-\beta \lambda}\right\} \frac{x^{p}}{s_{1}+s_{2}-p} \\
& =\frac{\lambda(1-\eta)^{p}}{\lambda-\eta}+\frac{(1-\beta)^{p} \lambda^{p}}{1-\beta \lambda} \operatorname{by}_{(3.23) .}
\end{aligned}
$$

Similarly,

$$
\limsup _{\delta \rightarrow 0}\|g+\lambda x\|_{p}^{p} \leq \frac{\lambda}{\lambda-\eta}+\frac{(\beta \lambda)^{p}}{1-\beta \lambda} .
$$

We proceed to a lower estimate of $\pi_{k}$. Note that if $w>1$ and $w_{1}=$ $w(x-\delta) /(x-w \delta)$, then

$$
1-w s \geq(1-s)^{w_{1}}, \quad s \in\left(0, \frac{\delta}{x}\right) .
$$

To see this, consider

$$
\frac{\log (1-w s)}{\log (1-s)}=\frac{\log (1-w s)-\log 1}{\log (1-s)-\log 1}
$$

by mean value theorem, there exists a $c \in(0, s)$,

$$
=\frac{-w /(1-w c)}{-1 /(1-c)}=\frac{w-1}{1-w c}+1 \leq \frac{w-1}{1-w \delta / x}+1=w_{1} .
$$

Therefore,

$$
\log (1-w s) \geq w_{1} \log (1-s)
$$

i.e.,

$$
1-w s \geq(1-s)^{w_{1}}
$$


To obtain a lower estimate of $\pi_{k}$, let

$$
\begin{aligned}
r_{1} & =\frac{x-\delta}{(1-\beta \lambda) x-\delta}, \quad r_{2}=\frac{\lambda(x-\delta)}{(\lambda-\eta) x-\lambda \delta}, \\
\pi_{k} & \geq \prod_{j=1}^{k}\left(1-\frac{\delta}{x+j \delta}\right)^{r_{1}}\left(1-\frac{\delta}{x+j \delta+\frac{\eta \delta}{\lambda-\eta}}\right)^{r_{2}} \\
& =\frac{x^{r_{1}+r_{2}}}{(x+k \delta)^{r_{1}+r_{2}}}\left(\frac{x+k \delta}{x+k \delta+\frac{\eta \delta}{\lambda-\eta}}\right)^{r_{2}}\left(\frac{x+\frac{\eta \delta}{\lambda-\eta}}{x}\right)^{r_{2}} \\
& \geq \frac{x^{r_{1}+r_{2}}}{(x+k \delta)^{r_{1}+r_{2}}} H_{2}(\delta),
\end{aligned}
$$

where

$$
H_{2}(\delta)=\left[\frac{x+k \delta}{x+k \delta+\frac{\eta \delta}{\lambda-\eta}}\right]^{r_{2}} \rightarrow 1 \quad \text { as } \delta \rightarrow 0 .
$$

Furthermore, as $\delta \rightarrow 0, r_{1} \rightarrow 1 /(1-\beta \lambda)$ and $r_{2} \rightarrow \lambda /(\lambda-\eta)$. Therefore,

$$
\begin{aligned}
\liminf _{\delta \rightarrow 0}\|f\|_{p}^{p} & \geq \liminf _{\delta \rightarrow 0}\left\{\frac{\lambda(1-\eta)^{p}}{\lambda-\eta}+\frac{(1-\beta)^{p} \lambda^{p}}{1-\beta \lambda}\right\} \sum_{k=1}^{\infty} \frac{\delta x^{r_{1}+r_{2}} H_{2}(\delta)}{(x+k \delta)^{r_{1}+r_{2}-p+1}} \\
& =\liminf _{\delta \rightarrow 0}\left\{\frac{\lambda(1-\eta)^{p}}{\lambda-\eta}+\frac{(1-\beta)^{p} \lambda^{p}}{1-\beta \lambda}\right\} \int_{0}^{\infty} \frac{\delta x^{r_{1}+r_{2}}}{(x+t \delta)^{r_{1}+r_{2}-p+1}} d t \\
& =\frac{\lambda(1-\eta)^{p}}{\lambda-\eta}+\frac{(1-\beta)^{p} \lambda^{p}}{1-\beta \lambda}
\end{aligned}
$$

i.e.,

$$
\lim _{\delta \rightarrow 0}\|f\|_{p}^{p}=\frac{\lambda(1-\eta)^{p}}{\lambda-\eta}+\frac{(1-\beta)^{p} \lambda^{p}}{1-\beta \lambda}
$$

Similarly

$$
\lim _{\delta \rightarrow 0}\|g+\lambda x\|_{p}^{p}=\frac{\lambda}{\lambda-\eta}+\frac{(\beta \lambda)^{p}}{1-\beta \lambda}
$$

so

$$
\begin{gathered}
\lim _{x \rightarrow 0} \lim _{\delta \rightarrow 0}\|f\|_{p}^{p}=\frac{\lambda(1-\alpha)^{p}}{\lambda-\alpha}+\frac{(1-\beta)^{p} \lambda^{p}}{1-\beta \lambda} \\
=\frac{1}{k_{p}} \lim _{x \rightarrow 0} \lim _{\delta \rightarrow 0}\|g+\lambda x\|_{p}^{p}
\end{gathered}
$$

by Lemma 3.4(i) since $\beta>0$ for $p_{0}<p<\infty$

$$
=\frac{1}{k_{p}} \lim _{x \rightarrow 0} \lim _{\delta \rightarrow 0}\|g\|_{p}^{p},
$$

i.e., $c_{p}^{p}(1.5) \geq k_{p}$.

Case (2). $2<p \leq p_{0}$. Fix $x>0$ and choose $\delta>0$ such that $\delta \ll x$. For all $k \geq 1$, define $\xi_{k}, \gamma_{k}, \pi_{k}, d_{k}$ and $\theta_{k}$ as in Case (1). Similar calculation as in Case (1) shows that $k_{p} \leq c_{p}^{p}(1.5)$. This completes the proof, apart from the lemmas, of Theorem 3.3. 
Remark. The martingale $f$ described in Part II of the proof of Theorem 3.3 is actually constructed from a zigzag martingale by (2.3). Here we provide a brief geometrical description of this zigzag martingale. Let $x>0$, choose $\eta \in(\alpha, \lambda)$ by (3.23). The zigzag martingale first starts at $(x, \lambda x)$, it then moves vertically either down to the point $(x, \eta x)$ where it will stop or up to the point $(x, \lambda(x+\delta))$. From the point $(x, \lambda(x+\delta))$, the zigzag martingale moves horizontal either to the left to the point $(\beta \lambda(x+\delta), \lambda(x+\delta))$ where it will stop or to the right to the point $(x+\delta, \lambda(x+\delta))$. The pattern of movement is then repeated.

\section{ASYMPTOTIC RESULTS AND REMARKS}

We make use of a result of Burkholder in [3] and triangle inequality to obtain Theorem 4.1. For $1<p<\infty$,

$$
\max \left\{1, \frac{p^{*}}{2}-1\right\} \leq c_{p}(1.5) \leq \frac{1}{2} p^{*},
$$

where $p^{*}=\max \{p, p /(p-1)\}$.

Proof. Let $d=\left(d_{1}, d_{2}, \ldots\right)$ be a martingale difference sequence and $\theta_{k} \in$ $\{0,1\}, k \geq 1$. Let $\varepsilon_{k}=2 \theta_{k}-1$. Then $\varepsilon_{k} \in\{1,-1\}$ and

$$
\begin{aligned}
\left\|\sum_{k=1}^{n} \theta_{k} d_{k}\right\| & =\frac{1}{2}\left\|\sum_{k=1}^{n} d_{k}+\sum_{k=1}^{n} \varepsilon_{k} d_{k}\right\|_{p} \\
& \leq \frac{1+c_{p}(1.4)}{2}\left\|\sum_{k=1}^{n} d_{k}\right\|_{p} .
\end{aligned}
$$

Therefore, $c_{p}(1.5) \leq\left(1+c_{p}(1.4)\right) / 2=\frac{1}{2} p^{*}$.

Similarly, let $\varepsilon_{k} \in\{1,-1\}$ and define $\theta_{k}=\left(\varepsilon_{k}+1\right) / 2 \in\{0,1\}$. Then

$$
\begin{aligned}
\left\|\sum_{k=1}^{n} \varepsilon_{k} d_{k}\right\|_{p} & =\left\|2 \sum_{k=1}^{n} \theta_{k} d_{k}-\sum_{k=1}^{n} d_{k}\right\|_{p} \\
& \leq\left[2 c_{p}(1.5)+1\right]\left\|\sum_{k=1}^{n} d_{k}\right\|_{p} .
\end{aligned}
$$

Therefore, $c_{p}(1.4)-1 \leq 2 c_{p}(1.5)$. On the other hand, $c_{p}(1.5) \geq 1$, Theorem 4.1 follows immediately.

We assume that $t_{p}=\sum_{k=0}^{\infty} a_{k} p^{-k}, p_{0}<p<\infty$. By (3.10) and (3.8), we rewrite (3.11) as

$$
\left\{t_{p}+\left[p-2-(p-1) t_{p}\right]^{1 /(p-1)}\right\}^{-1}=\frac{1}{E\left(t_{p}\right)}-\frac{p-2-p t_{p}}{(p-1)\left(1-t_{p}\right)^{2}} .
$$

From Theorem 3.3, we have $a_{0}=1, a_{1}=-2$. Substituting $t_{p}=\sum_{k=0}^{\infty} a_{k} p^{-k}$ into (4.1) and equating coefficients of $p^{-k}, k=0,1,2, \ldots$, we obtain 
Theorem 4.2. Assuming $t_{p}=\sum_{k=0}^{\infty} a_{k} p^{-k}, p_{0}<p<\infty$, then

$$
\begin{aligned}
t_{p}= & 1-\frac{2}{p}-\frac{2(1-\gamma)}{(1+\gamma)} \frac{1}{p^{2}}-\frac{4 \gamma(3-\gamma)(1-\gamma)}{(1+\gamma)^{3}} \frac{1}{p^{3}} \\
& +\frac{4 \gamma\left(-9 \gamma^{4}+56 \gamma^{3}-186 \gamma^{2}+120 \gamma-13\right)}{3(1+\gamma)^{5}} \frac{1}{p^{4}}+\cdots,
\end{aligned}
$$

where $\gamma=e^{-2}$.

We substitute these known coefficients into (3.12), we have

Theorem 4.3. For $p_{0}<p<\infty$,

$$
c_{p}(1.5)=\frac{p}{2}+\frac{1}{2} \log \left(\frac{1+\gamma}{2}\right)+\frac{\alpha_{2}}{p}+\cdots,
$$

where $\gamma=e^{-2}$ and

$$
\alpha_{2}=\left[\frac{1}{2} \log \left(\frac{1+\gamma}{2}\right)\right]^{2}+\frac{1}{2} \log \left(\frac{1+\gamma}{2}\right)-2\left(\frac{\gamma}{1+\gamma}\right)^{2} .
$$

Remark. That $a_{2}=-2(1-\gamma) /(1+\gamma)$ can also be derived from Theorem 4.1 and the fact that $t_{p}=1-2 / p+a_{2} / p^{2}+\cdots$. Indeed, Theorem 4.1 implies that $c_{p}(1.5)=p / 2+0(1)$. Substitute, $t_{p}=1-2 / p+a_{2} / p^{2}+O\left(1 / p^{3}\right)$ into (3.12) and by Theorem 3.3, we have $c_{p}(1.5)=p(1+\gamma) /\left(4+(1+\gamma) a_{2}\right)+O(1)$. this implies $a_{2}=-2(1-\gamma) /(1+\gamma)$.

\section{Proof of LemMas}

Proof of Lemma 3.1. We will give a sketch of the proof. For details, see [6].

Case 1. $p_{0}<p<\infty$. Define $a(t)=A(t) /(1-t)^{2}$ and $b(t)=B(t) /(1-t)^{2}$.

Step 1. We shall show that $a(t)$ is increasing on $I_{p}, b(t)$ is decreasing on $I_{p}$ and that they are both positive on $I_{p}$.

We note that $E(t)$ is positive and decreasing on $(0,1)$. Convexity of $E(t)$ on $(0,1)$ and $E(1)=0$ imply that $E(t) /(1-t)$ is a positive decreasing function on $(0,1)$. Note that

$$
\begin{aligned}
& a(t)=p-1-\frac{[(p-2)-p t]}{(1-t)} \cdot \frac{E(t)}{1-t}, \quad \text { and } \\
& b(t)=(p-1) E(t)-t a(t) .
\end{aligned}
$$

Now $a(t)$ is increasing on $(0,(p-2) / p)$ because $[(p-2)-p t] /(1-t)$ and $E(t) /(1-t)$ are both positive and decreasing on $(0,(p-2) / p)$. By direct verification, we have $a((p-2) / p)>0$ and $b((p-2) / p)>0$.

(i) When $p_{0}<p \leq 3, a(0) \geq 0$. Therefore $a(t)>0$ on $(0,(p-2) / p)$. It follows from $(5.2)$ that $b(t)$ is decreasing on $(0,(p-2) / p)$ and hence positive on $I_{p}$.

(ii) When $3<p<\infty$. There exists a unique $t_{1} \in(0,(p-2) / p)$ such that $a\left(t_{1}\right)=0$. Since $a((p-3) /(p-1))>0$, it implies that $a(t)>0$ on $I_{p}$. It follows from (5.2) that $b(t)$ is decreasing on $I_{p}$ and hence positive on $I_{p}$.

Step 2. Existence of a solution to (3.11). Define

$$
\Delta(t)=(p-2)-(p-1) t-\left[\frac{B(t)}{A(t)}\right]^{p-1}, \quad t \in I_{p} .
$$


(i) When $p_{0}<p \leq 3$, we can verify that

$$
\Delta(0)=p-2-\left[\frac{(p-2)(p-1)}{-p^{2}+5 p-5}\right]^{p-1}<0 .
$$

(This explains equation (3.2).)

(ii) When $3<p<\infty$, we have $A((p-3) /(p-1))<B((p-3) /(p-1))$, therefore $\Delta((p-3) /(p-1))<0$. Now $\Delta(t)$ is continuous and $\Delta((p-2) / p)>0$. Existence of a solution to (3.11) follows.

Step 3. Uniqueness of solution to (3.11). For $3<p<\infty$, we first show that $a(t)$ is convex on $(0,1)$. Differentiate $(5.1)$ twice and let $M(t)$ be the numerator. When $4<p<\infty, M^{\prime \prime}(t)<0$ on $(0, \tilde{s})$, where $\tilde{s}=$ $(p-4)(p-2) / p^{2} ; M^{\prime \prime}(t)>0$ on $(\tilde{s}, 1)$. Computing $M^{\prime}(t)$ at 0 and 1 , we see that $M^{\prime}(t)<0$ on $(0,1)$. Therefore, $M(t)>M(1)=0$. When $3<p \leq 4$, $M^{\prime \prime}(t)>0$ on $(0,1)$, therefore $M^{\prime}(t)<M^{\prime}(1)=0$ and this implies that $M(t)>0$ on $(0,1)$. Now we deduce that $[p-2-(p-1) t](a(t))^{p-1}$ is increasing on $I_{p}$. Its derivative equals $(a(t))^{p-2}\left\{[p-2-p t] a^{\prime}(t)+t a^{\prime}(t)-a(t)\right\}$ which is positive because $t a^{\prime}(t)-a(t)$ is increasing, therefore $t a^{\prime}(t)-a(t)>t_{1} a^{\prime}\left(t_{1}\right)>0$ for $t \in\left(t_{1},(p-2) / p\right)$. Uniqueness of solution to (3.11) follows immediately since $(b(t))^{p-1}$ is decreasing. For $p_{0}<p \leq 3$, we show that $b(t) / a(t)$ is convex on $\left(0, t_{2}\right)$. Uniqueness of solution to (3.11) follows because $[p-2-(p-1) t]$ is linear and $(b(t) / a(t))^{p-1}$ is convex. To show that $b(t) / a(t)$ is convex, we differentiate it twice and rewrite the numerator as

$$
N(t)=a(t)\left[a(t) b^{\prime \prime}(t)-2 a^{\prime}(t) b^{\prime}(t)\right]+\left(-a^{\prime \prime}(t)\right) a(t) b(t)+2 b(t) a^{\prime}(t) .
$$

Since $a^{\prime \prime}(t)<0$ in this case. To show that $N(t)$ is positive, it suffices to show that $\Delta_{1}(t)=a(t) b^{\prime \prime}(t)-2 a^{\prime}(t) b^{\prime}(t) \geq 0$. Recall $b(t)=(p-1) E(t)-t a(t)$ from (5.2), $\Delta_{1}(t)=(p-1) a(t) E^{\prime \prime}(t)+t a(t)\left[-a^{\prime \prime}(t)\right]+2(p-1) a^{\prime}(t)\left(-E^{\prime}(t)\right)+t\left(a^{\prime}(t)\right)^{2}$. Each of these four terms is nonnegative on $I_{p}$. This completes the proof of Lemma 3.1 for Case 1.

Case 2. $2<p \leq p_{0}$. We will be brief in the proof. We are able to show that $A(t)$ and $B(t)$ are positive on $I_{p}$, and by (3.5), $\Delta(0)>0$ (see (5.3)). It can be shown that $\Delta(-(3-p) / 2 p)<0$, existence of a solution follows. Lastly we can show that $[p-2-(p-1) t](B(t) / A(t))^{p-1}$ is decreasing and uniqueness is proved.

This completes the proof of Lemma 3.1.

Proof of Lemma 3.4. We note that $\left(p-1-p t_{p}\right),\left[p-2-(p-1) t_{p}\right], D\left(t_{p}\right)$ and $E\left(t_{p}\right)$ are positive, so $\alpha>0$. Also, $\lambda-\alpha=\left(1-t_{p}\right) E\left(t_{p}\right) / D\left(t_{p}\right)>0$ and $1-\beta \lambda=1-t_{p}>0$. When $p_{0}<p<\infty, t_{p}>0$ so $\beta>0$. Since $\beta \lambda=t_{p}<1$, this implies $\lambda<\beta^{-1}$. When $2<p \leq p_{0}, t_{p} \leq 0$ so $\beta \leq 0$. This completes the proof of (iii) and (iii') .

It is easy to show (ii) by (3.19) to (3.21).

From (3.7) and (3.9), we see readily that the left-hand side of (i) equals $D\left(t_{p}\right) /\left(1-t_{p}\right)$. By definition of $A(t)$ in (3.8), we have

$$
\frac{\lambda(1-\alpha)^{p}}{\lambda-\alpha}=\frac{\left[p-1-p t_{p}\right]}{\left(1-t_{p}\right)}\left[\frac{A\left(t_{p}\right)}{D\left(t_{p}\right)}\right]^{p} .
$$


From $\left(3.10^{\prime}\right)$,

$$
\frac{(1-\beta)^{p} \lambda^{p}}{\lambda-\alpha}=\frac{1}{\left(1-t_{p}\right)} \cdot\left[\frac{B\left(t_{p}\right)}{D\left(t_{p}\right)}\right]^{p} .
$$

Since $t_{p}$ is a solution to (3.11), the right-hand side of (i) can be simplified as

$$
\frac{\left.\left(p-1-p t_{p}\right) A\left(t_{p}\right)+[p-2-p-1) t_{p}\right] B\left(t_{p}\right)}{(p-1)\left(1-t_{p}\right)^{3}}
$$

which can be further simplified as $D\left(t_{p}\right) /\left(1-t_{p}\right)$ by $\left(3.10^{\prime}\right),(3.8)$ and (3.9). This completes the proof of (i) and hence Lemma 3.4.

Let

$$
\begin{aligned}
& A=1-k_{p}(1-\alpha)^{p}=v(x, \alpha x)|x|^{-p}, \\
& B=|\beta|^{p}-k_{p}(1-\beta)^{p}=v(\beta y, y)|y|^{-p} .
\end{aligned}
$$

To prove Lemma 3.2, we need the following identities and inequalities which are grouped under the following lemma.

Lemma 5.1. For $2<p<\infty$, we have

(i) $1-\alpha=\frac{A\left(t_{p}\right)}{D\left(t_{p}\right)}>0$,

(ii) $A=\frac{\left[(p-2)-p t_{p}\right]}{(p-1)\left(1-t_{p}\right)^{2}} E\left(t_{p}\right)>0$,

(iii) $1-k_{p}(1-\alpha)^{p-2}<0$,

(iv) $\omega-A=\frac{p E\left(t_{p}\right)}{(p-1)\left(1-t_{p}\right)}>0$,

(v) $\frac{\omega-A}{\lambda-\alpha}=p k_{p}(1-\alpha)^{p-1}$,

(vi) $p A-\frac{\alpha(\omega-A)}{\lambda-\alpha}=p\left[1-k_{p}(1-\alpha)^{p-1}\right]$,

(vii) $p A+(p-2)(\omega-A)-\frac{2 \alpha(\omega-A)}{\lambda-\alpha}=0$,

(viii) $1-\beta=\frac{B\left(t_{p}\right)}{\left[(p-1)-p t_{p}\right] E\left(t_{p}\right)}>0$,

(ix) $\omega-B \lambda^{p}=\frac{p\left[(p-1)-p t_{p}\right]}{(p-1)\left(1-t_{p}\right)} E\left(t_{p}\right)>0$,

(x) $\quad p\left[\operatorname{sgn}(\beta)|\beta|^{p-1}+k_{p}(1-\beta)^{p-1}\right]=\frac{\lambda}{1-\beta \lambda}\left[\frac{\omega}{\lambda^{p}}-B\right]$,

(xi) $\quad-p k_{p}(1-\beta)^{p-1}=p B-\frac{\beta \lambda}{1-\beta \lambda}\left[\frac{\omega}{\lambda^{p}}-B\right]$,

(xii) $p B+(p-2)\left[\frac{\omega}{\lambda^{p}}-B\right]-\frac{2 \beta \lambda}{1-\beta \lambda}\left[\frac{\omega}{\lambda^{p}}-B\right]=0$,

(xiii) $\frac{\omega-B \lambda^{p}}{1-\beta \lambda}=p A-\frac{\alpha(\omega-A)}{\lambda-\alpha}+(p-1)(\omega-A)$,

(xiv) $p B \lambda^{p-1}+(p-1)\left[\frac{\omega}{\lambda^{p}}-B\right] \lambda^{p-1}-\frac{\beta \lambda}{1-\beta \lambda}\left[\frac{\omega}{\lambda^{p}}-B\right] \lambda^{p-1}=\frac{\omega-A}{\lambda-\alpha}$,

(xv) $|\beta|^{p-2}-k_{p}(1-\beta)^{p-2}<0$. 
Proof of Lemma 5.1. From (3.19), (3.8) and (3.9) we get (i). By (i), (3.12) and (3.8), we get (ii). Using (i), (3.12) and (3.9), we obtain

$$
1-k_{p}(1-\alpha)^{p-1}=1-\frac{D\left(t_{p}\right)}{(p-1)\left(1-t_{p}\right)^{2}}=\frac{-t_{p} E\left(t_{p}\right)}{(p-1)\left(1-t_{p}\right)^{2}}<0 .
$$

Therefore, $1<k_{p}(1-\alpha)^{p-1}<k_{p}(1-\alpha)^{p-2}$, so (iii) is proved. From (ii) and (3.22), we prove (iv). From (iv), (3.19) and (3.21), both sides of (v) equal $D\left(t_{p}\right) /(p-1)\left(1-t_{p}\right)^{2}$, so (v) follows.

Now,

$$
\begin{aligned}
p A-\frac{\alpha(\omega-A)}{\lambda-\alpha} & =p A-p \alpha k_{p}(1-\alpha)^{p-1} \quad \text { by }(\mathrm{v}) \\
& =p\left[1-k_{p}(1-\alpha)^{p}\right]-p \alpha k_{p}(1-\alpha)^{p-1} \text { by }(5.4) \\
& =p\left[1-k_{p}(1-\alpha)^{p-1}\right],
\end{aligned}
$$

which is (vi). From (ii), (iv) and (v), we can prove (vii). By (3.20) and $\left(3.10^{\prime}\right)$, we prove (viii).

To show (ix). From (viii), we obtain

$$
\begin{aligned}
\omega-B \lambda^{p}= & \frac{2\left[p-1-p t_{p}\right]}{(p-1)\left(1-t_{p}\right)^{2}} E\left(t_{p}\right)-\left|t_{p}^{p}\right|+\frac{\left[B\left(t_{p}\right)\right]^{p}}{(p-1)\left(1-t_{p}\right)^{2}\left[A\left(t_{p}\right)\right]^{p-1}} \\
= & \frac{2\left[p-1-p t_{p}\right]}{(p-1)\left(1-t_{p}\right)^{2}} E\left(t_{p}\right)+\left[p-1-p t_{p}\right]-D\left(t_{p}\right) \\
& +\frac{\left[p-2-(p-1) t_{p}\right]\left\{\left[p-1-p t_{p}\right] E\left(t_{p}\right)-t_{p} D\left(t_{p}\right)\right\}}{(p-1)\left(1-t_{p}\right)^{2}} \\
= & \frac{\left[p-1-p t_{p}\right]\left[p-(p-1) t_{p}\right] E\left(t_{p}\right)}{(p-1)\left(1-t_{p}\right)^{2}}-\frac{\left[p-1-p t_{p}\right]}{(p-1)\left(1-t_{p}\right)^{2}} D\left(t_{p}\right) \\
& +\left[p-1-p t_{p}\right] \\
= & \frac{\left[p-1-p t_{p}\right]\left[p-(p-1) t_{p}\right] E\left(t_{p}\right)}{(p-1)\left(1-t_{p}\right)^{2}}-\frac{\left[p-1-p t_{p}\right] t_{p} E\left(t_{p}\right)}{(p-1)\left(1-t_{p}\right)^{2}} \\
= & \frac{p\left[(p-1)-p t_{p}\right] E\left(t_{p}\right)}{(p-1)\left(1-t_{p}\right)}>0 .
\end{aligned}
$$

To show $(\mathbf{x})$. It is enough to show that

$$
\frac{\omega-B \lambda^{p}}{1-\beta \lambda}=p\left[\operatorname{sgn}(\beta)|\beta|^{p-1}+k_{p}(1-\beta)^{p-1}\right] \lambda^{p-1} \text {. }
$$

Now,

$$
\begin{aligned}
\text { R.H.S. } & =p\left\{\operatorname{sgn}\left(t_{p}\right)\left|t_{p}\right|^{p-1}+\frac{D\left(t_{p}\right)\left[(p-2)-(p-1) t_{p}\right]}{(p-1)\left(1-t_{p}\right)^{2}}\right\} \\
= & p\left\{E\left(t_{p}\right)-\left[(p-2)-(p-1) t_{p}\right]\right. \\
& \left.+\frac{D\left(t_{p}\right)\left[(p-2)-(p-1) t_{p}\right]}{(p-1)\left(1-t_{p}\right)^{2}}\right\} \\
= & p \frac{\left[(p-1)-p t_{p}\right] E\left(t_{p}\right)}{(p-1)\left(1-t_{p}\right)^{2}}=\text { L.H.S., so }(\mathrm{x}) \text { is verified. }
\end{aligned}
$$


Now,

$$
\begin{aligned}
B & =|\beta|^{p}-k_{p}(1-\beta)^{p} \\
& =\beta\left\{\operatorname{sgn}(\beta)|\beta|^{p-1}+k_{p}(1-\beta)^{p-1}\right\}-k_{p}(1-\beta)^{p-1} .
\end{aligned}
$$

Therefore, by $(\mathbf{x})$, we have,

$$
B=\frac{\beta \lambda}{p(1-\beta \lambda)}\left[\frac{\omega}{\lambda^{p}}-B\right]-k_{p}(1-\beta)^{p-1} .
$$

Multiplying throughout by $p$ and rearranging terms, we obtain (xi). Instead of verifying (xii), we will show that

$$
p B \lambda^{p}=\left[\frac{2 \beta \lambda}{1-\beta \lambda}-(p-2)\right]\left[\omega-B \lambda^{p}\right],
$$

which is equivalent to (xii). Now,

$$
\begin{aligned}
B \lambda^{p} & =\left[B \lambda^{p}-\omega\right]+\omega \\
& =\frac{-p\left[(p-1)-p t_{p}\right] E\left(t_{p}\right)}{(p-1)\left(1-t_{p}\right)}+\frac{2\left[(p-1)-p t_{p}\right] E\left(t_{p}\right)}{(p-1)\left(1-t_{p}\right)^{2}} \text { by (ix) } \\
& =\frac{p\left[(p-1)-p t_{p}\right] E\left(t_{p}\right)}{(p-1)\left(1-t_{p}\right)}\left\{\frac{2}{p\left(1-t_{p}\right)}-1\right\} \\
& =\frac{p\left[(p-1)-p t_{p}\right] E\left(t_{p}\right)}{(p-1)\left(1-t_{p}\right)}\left\{\frac{2 t_{p}}{p\left(1-t_{p}\right)}-\left(1-\frac{2}{p}\right)\right\} .
\end{aligned}
$$

Since $\beta \lambda=t_{p}$ and by (ix), so

$$
B \lambda^{p}=\left\{\frac{2 \beta \lambda}{p(1-\beta \lambda)}-\left(1-\frac{2}{p}\right)\right\}\left[\omega-B \lambda^{p}\right],
$$

this implies (xii). Rewriting (vii) and (xii), they become

$$
\frac{\lambda A}{\lambda-\alpha}=\left[\frac{\lambda}{\lambda-\alpha}-\frac{p}{2}\right] \omega \text { and } \frac{B \lambda^{p}}{1-\beta \lambda}=\left[\frac{1}{1-\beta \lambda}-\frac{p}{2}\right] \omega \text {. }
$$

Therefore,

$$
\frac{\lambda A}{\lambda-\alpha}+\frac{B \lambda^{p}}{1-p \lambda}=\left[\frac{\lambda}{\lambda-\alpha}+\frac{1}{1-\beta \lambda}-p\right] \omega
$$

which is equivalent to (xiii).

$$
\begin{aligned}
\text { L.H.S. of (xiv) }= & \frac{2 \beta \lambda}{1-\beta \lambda}\left[\frac{\omega}{\lambda^{p}}-B\right] \lambda^{p-1}-(p-2)\left[\frac{\omega}{\lambda^{p}}-B\right] \lambda^{p-1} \\
& +(p-1)\left[\frac{\omega}{\lambda^{p}}-B\right] \lambda^{p-1}-\frac{\beta \lambda}{1-\beta \lambda}\left[\frac{\omega}{\lambda^{p}}-B\right] \lambda^{p-1} \quad \text { by (xii) } \\
= & \left\{1+\frac{\beta \lambda}{1-\beta \lambda}\right\}\left[\frac{\omega}{\lambda^{p}}-B\right] \lambda^{p-1} \\
= & \frac{p}{(p-1)} \frac{D\left(t_{p}\right)}{\left(1-t_{p}\right)^{2}} \quad \text { by (ix) and (3.21). }
\end{aligned}
$$

By (iv), (3.19) and (3.21), R.H.S. of (xiv) $=p D\left(t_{p}\right) /(p-1)\left(1-t_{p}\right)^{2}$, so we have proved (xiv). 
It remains to show $(\mathrm{xv})$. It is enough to verify $k_{p}((1-\beta) /|\beta|)^{p-2}>1$. Now, by (viii), (3.20), (3.12) and (3.11), we have

$$
k_{p}\left(\frac{1-\beta}{|\beta|}\right)^{p-1}-1=\frac{\left[p-2-(p-1) t_{p}\right]\left[D\left(t_{p}\right)\right]^{2}}{\left|t_{p}\right|^{p-2}(p-1)\left(1-t_{p}\right)^{2} B\left(t_{p}\right)}-1 .
$$

Therefore, it suffices to show that

$$
\left[(p-2)-(p-1) t_{p}\right]\left[D\left(t_{p}\right)\right]^{2}-(p-1)\left(1-t_{p}\right)^{2}\left|t_{p}\right|^{p-2} B\left(t_{p}\right)>0 .
$$

Using $\left(3.10^{\prime}\right)$, we can rewrite the

L. H. S. $=\left\{\left[(p-2)-(p-1) t_{p}\right] D\left(t_{p}\right)+(p-1)\left(1-t_{p}\right)^{2} \operatorname{sgn}\left(t_{p}\right)\left|t_{p}\right|^{p-1}\right\} D\left(t_{p}\right)$

$$
-(p-1)\left(1-t_{p}\right)^{2}\left|t_{p}\right|^{p-2}\left[(p-1)-p t_{p}\right] E\left(t_{p}\right) \text {. }
$$

We simplify the expression inside \{\} :

$$
\begin{aligned}
& {\left[(p-2)-(p-1) t_{p}\right] D\left(t_{p}\right)+(p-1)\left(1-t_{p}\right)^{2}\left[E\left(t_{p}\right)-(p-2)+(p-1) t_{p}\right]} \\
& \quad=\left[(p-2)-(p-1) t_{p}\right]\left\{D\left(t_{p}\right)-(p-1)\left(1-t_{p}\right)^{2}\right\}+(p-1)\left(1-t_{p}\right)^{2} E\left(t_{p}\right) \\
& \quad=\left[(p-1)-p t_{p}\right] E\left(t_{p}\right) \text { by }(3.9) .
\end{aligned}
$$

So,

L. H. S. $=\left[(p-1)-p t_{p}\right] E\left(t_{p}\right) D\left(t_{p}\right)-(p-1)\left(1-t_{p}\right)^{2}\left|t_{p}\right|^{p-2}\left[(p-1)-p t_{p}\right] E\left(t_{p}\right)$

$$
\begin{aligned}
& =\left[(p-1)-p t_{p}\right] E\left(t_{p}\right)\left\{D\left(t_{p}\right)-(p-1)\left(1-t_{p}\right)^{2}\left|t_{p}\right|^{p-2}\right\} \\
& >\left[(p-1)-p t_{p}\right] E\left(t_{p}\right)(p-1)\left(1-t_{p}\right)^{2}\left(1-\left|t_{p}\right|^{p-2}\right) \text { by }(3.9) \\
& >0 .
\end{aligned}
$$

So $(\mathbf{x v})$ is established and this concludes the proof of Lemma 5.1.

Proof of Lemma 3.2. Define

$$
\begin{aligned}
& \Omega_{1}=\left\{(x, y) \in \mathbf{R}^{2}: y>0, y<\alpha x\right\}, \\
& \Omega_{2}=\left\{(x, y) \in \mathbf{R}^{2}: y>0, \alpha x<y<\lambda x\right\}, \\
& \Omega_{3}=\left\{(x, y) \in \mathbf{R}^{2}: y>0, \beta y<x<\lambda^{-1} y\right\}, \\
& \boldsymbol{\Omega}_{4}=\left\{(x, y) \in \mathbf{R}^{2}: y>0, x<\beta y\right\} .
\end{aligned}
$$

Let $u(x, y)$ be the continuous function from $\mathbf{R}^{2}$ to $\mathbf{R}$ satisfying $u(x, y)=$ $u(-x,-y)$, and

$$
u(x, y)= \begin{cases}v(x, y), & (x, y) \in \Omega_{1} \cup \Omega_{4}, \\ A x^{p}+\frac{y-\alpha x}{\lambda-\alpha}(\omega-A) x^{p-1}, & (x, y) \in \Omega_{2}, \\ B y^{p}+\frac{\lambda(x-\beta y)}{1-\beta \lambda}\left[\frac{\omega}{\lambda^{p}}-B\right] y^{p-1}, & (x, y) \in \Omega_{3} .\end{cases}
$$

The bounds on $u, u_{x}$ and $u_{y}$, and $u(0,0)=0$ can be verified readily.

Step 1 . To show that $u$ is concave in $y$ on $\bigcup_{i=1}^{4} \Omega_{i}$. On $\Omega_{1} \cup \Omega_{4}, u_{y y}(x, y)=$ $-p(p-1) k_{p}|x-y|^{p-2}<0$, therefore $u$ is concave in $y$. On $\Omega_{2}, u_{y y} \equiv 0$, hence $u$ is concave in $y$. On $\Omega_{3}$,

$$
\begin{aligned}
u_{y y}(x, y)= & p(p-1) B y^{p-2}-\frac{2(p-1) \beta \lambda}{1-\beta \lambda}\left[\frac{\omega}{\lambda^{p}}-B\right] y^{p-2} \\
& +(p-1)(p-2) \frac{\lambda(x-\beta y)}{1-\beta \lambda}\left[\frac{\omega}{\lambda^{p}}-B\right] y^{p-3} .
\end{aligned}
$$


Now,

$$
\begin{aligned}
u_{y y}\left(\left(\frac{y}{\lambda}\right)-, y\right) & =(p-1)\left\{p B+(p-2)\left[\frac{\omega}{\lambda^{p}}-B\right]-\frac{2 \beta \lambda}{1-\beta \lambda}\left[\frac{\omega}{\lambda^{p}}-B\right]\right\} y^{p-2} \\
& =0 \text { by Lemma } 5.1 \text { (xii) }
\end{aligned}
$$

So,

$$
\begin{aligned}
u_{y y}(x, y) & =u_{y y}(x, y)-u_{y y}\left(\left(\frac{y}{\lambda}\right)-, y\right) \\
& =\frac{(p-1)(p-2)(\lambda x-y)}{1-\beta \lambda}\left[\frac{\omega}{\lambda^{p}}-B\right] y^{p-3} \\
& <0 \text { by Lemma 5.1(ix) and that } y>\lambda x \text { on } \Omega_{3} .
\end{aligned}
$$

Step 2. To show that $u$ is concave in $x$ on $\bigcup_{i=1}^{4} \Omega_{i}$. On $\Omega_{1}$,

$$
\begin{aligned}
u_{x x}(x, y) & =p(p-1)\left[x^{p-2}-k_{p}(x-y)^{p-2}\right] \\
& <p(p-1)\left[1-k_{p}(1-\alpha)^{p-2}\right] x^{p-2}<0
\end{aligned}
$$

by Lemma 5.1 (iii).

On $\Omega_{4}$,

$$
\begin{aligned}
u_{x x}(x, y) & =p(p-1)\left[|x|^{p-2}-k_{p}(y-x)^{p-2}\right] \\
& <p(p-1)\left[|\beta|^{p-2}-k_{p}(1-\beta)^{p-2}\right] y^{p-2} \\
& <0 \text { by Lemma } 5.1(\mathrm{xv}) .
\end{aligned}
$$

On $\Omega_{2}, \quad u_{x x}(x, y)=p(p-1) A x^{p-2}-\frac{2(p-1) \alpha(\omega-A)}{\lambda-\alpha} x^{p-2}$

$$
+(p-1)(p-2) \frac{y-\alpha x}{\lambda-\alpha}(\omega-A) x^{p-3}
$$

Since

$$
u_{x x}(x, \lambda x-)=(p-1)\left\{p A+(p-2)(\omega-A)-\frac{2 \alpha(\omega-A)}{\lambda-\alpha}\right\} x^{p}=0
$$

so

$$
\begin{aligned}
u_{x x}(x, y) & =u_{x x}(x, y)-u_{x x}(x, \lambda x-) \\
& =\frac{(p-1)(p-2)(\omega-A)}{\lambda-\alpha}(y-\lambda x) x^{p-3}<0,
\end{aligned}
$$

by Lemma 5.1 (iv) and the fact that $y<\lambda x$ on $\Omega_{2}$. The function, $u$, is affine in $x$ on $\Omega_{3}$, so $u$ is concave in $x$ on $\bigcup_{i=1}^{4} \Omega_{i}$.

Step 3. To show that the first derivatives match up at the boundaries of the regions. At $y=\alpha x$, we have

$$
\begin{aligned}
u_{x}(x, \alpha x+) & =\left[p A-\frac{\alpha}{\lambda-\alpha}(\omega-A)\right] x^{p-1} \\
& =p\left[1-k_{p}(1-\alpha)^{p-1}\right] x^{p-1}=u_{x}(x, \alpha x-)
\end{aligned}
$$

from Lemma 5.1(vi). Also, we have

$$
\begin{aligned}
u_{y}(x, \alpha x+) & =\frac{\omega-A}{\lambda-\alpha} x^{p-1} \\
& =p k_{p}(1-\alpha)^{p-1} x^{p-1}=u_{y}(x, \alpha x-) \quad \text { by Lemma } 5.1(\mathrm{v})
\end{aligned}
$$


At $y=\lambda x$,

$$
\begin{aligned}
u_{x}(x, \lambda x+) & =\frac{\omega-B \lambda^{p}}{1-\beta \lambda} x^{p-1} \\
& =\left[p A-\frac{\alpha(\omega-A)}{\lambda-\alpha}+(p-1)(\omega-A)\right] x^{p-1}=u_{x}(x, \lambda x-)
\end{aligned}
$$

from Lemma 5.1(xiii); and

$$
\begin{aligned}
u_{y}(x, \lambda x+) & =\left\{p B \lambda^{p-1}+(p-1)\left[\frac{\omega}{\lambda^{p}}-B\right] \lambda^{p-1}-\frac{\beta \lambda}{1-\beta \lambda}\left[\frac{\omega}{\lambda^{p}}-B\right] \lambda^{p-1}\right\} x^{p-1} \\
& =\frac{\omega-A}{\lambda-\alpha} x^{p-1}=u_{y}(x, \lambda x-)
\end{aligned}
$$

from Lemma 5.1(xiv). At $x=\beta y$, we get

$$
\begin{aligned}
u_{x}(\beta y-, y) & =p\left[\operatorname{sgn}(\beta)|\beta|^{p-1}+k_{p}(1-\beta)^{p-1}\right] y^{p-1} \\
& =\frac{\lambda}{1-\beta \lambda}\left[\frac{\omega}{\lambda^{p}}-B\right] y^{p-1}=u_{x}(\beta y+, y)
\end{aligned}
$$

from Lemma 5.1(x); and

$$
\begin{aligned}
u_{y}(\beta y-, y) & =-p k_{p}(1-\beta)^{p-1} y^{p-1} \\
& =\left\{p B-\frac{\beta \lambda}{1-\beta \lambda}\left[\frac{\omega}{\lambda^{p}}-B\right]\right\} y^{p-1}=u_{y}(\beta y+, y)
\end{aligned}
$$

from Lemma 5.1(xi).

Step 4. To show that $\lambda<1$. Since

$$
\begin{aligned}
1-\lambda & =\left\{D\left(t_{p}\right)-\left[p-1-p t_{p}\right] E\left(t_{p}\right)\right\} / D\left(t_{p}\right) \\
& =\left(1-t_{p}\right)^{2}\left\{a\left(t_{p}\right)-\frac{E\left(t_{p}\right)}{1-t_{p}}\right\} / D\left(t_{p}\right),
\end{aligned}
$$

it is enough to show that $a\left(t_{p}\right)-E\left(t_{p}\right) /\left(1-t_{p}\right)>0$. From Step 1 of the proof of Lemma 3.1, we see that $a(t)$ and $E(t) /(1-t)$ are increasing and decreasing functions respectively. Therefore, $\psi(t)=a(t)-E(t) /(1-t)$ is an increasing function. For $p_{0} \leq p \leq 3, \psi(0)=a(0)-E(0)=A(0)-E(0)=(3-p)(p-1) \geq$ 0 , therefore $\psi\left(t_{p}\right)>0$; for $3<p<\infty$,

$$
\psi\left(\frac{p-3}{p-1}\right)=-(p-1)\left[\frac{p-3}{p-1}\right]^{p-1}<0
$$

and

$$
\psi\left(\frac{p-2}{p}\right)=\frac{p}{2}\left[1-\left(\frac{p-2}{p}\right)^{p-1}\right]>0 \text {. }
$$

Therefore, there exists a unique $s_{p} \in I_{p}$ such that $\psi\left(s_{p}\right)=0$ and $\psi(t)>0$ on $\left(s_{p},(p-2) / p\right)$. So, $\psi\left(t_{p}\right)>0$ follows if we can prove that $s_{p}<t_{p}$. This can be reduced to proving

$$
\left[p-2-(p-1) s_{p}\right]-\left(b\left(s_{p}\right) / a\left(s_{p}\right)\right)^{p-1}<0 .
$$

As $\psi\left(s_{p}\right)=0$, it implies $\left(1-s_{p}\right) a\left(s_{p}\right)=E\left(s_{p}\right)$. Using this and (5.2), we get $b\left(s_{p}\right) / a\left(s_{p}\right)=p-1-p s_{p}>1$. Therefore,

$$
\left(\frac{b\left(s_{p}\right)}{a\left(s_{p}\right)}\right)^{p-1}>\frac{b\left(s_{p}\right)}{a\left(s_{p}\right)}=p-1-p s_{p}>(p-2)-(p-1) s_{p} .
$$


So, (5.7) follows and this finishes Step 4.

Step 5. To verify that $u(x, y) \geq v(x, y)$ on $\mathbf{R}^{2}$. By homogeneity and symmetry, it suffices to show that (i) $u(1, y) \geq v(1, y)$ for $y \in(\alpha, \lambda)$; and (ii) $u(x, 1) \geq v(x, 1)$ for $x \in\left(\beta, \lambda^{-1}\right)$.

Case (i). We observe that the graph of $u(1, y)$ for $\alpha<y<\lambda$ is part of the line tangent to the graph of the concave function $v(1, y)$ at $y=\alpha$. Therefore, $u(1, y)>v(1, y)$ for $\alpha<y<\lambda$.

Case (ii). Observe that again the graph of $u(x, 1)$ is tangent to the graph of the function $v(x, 1)$ at the point $x=\beta$. Since $u(1, \lambda)>v(1, \lambda)$ from case (i), $u\left(\lambda^{-1}, 1\right)>v\left(\lambda^{-1}, 1\right)$ by homogeneity. Let $m$ be the slope of the line defined by $u(x, 1)$ on $\left(\beta, \lambda^{-1}\right)$, i.e., $m=(\lambda /(1-\beta \lambda))\left[\omega / \lambda^{p}-B\right]$. Consider the function $V(x)=v(x, 1)$. We have,

$$
V^{\prime}(x)=p\left\{\operatorname{sgn}(x)|x|^{p-1}-\operatorname{sgn}(x-1)|x-1|^{p-1}\right\}
$$

and

$$
V^{\prime \prime}(x)=p(p-1)\left\{|x|^{p-2}-k_{p}|x-1|^{p-2}\right\} .
$$

There exist $\xi_{1}, \xi_{2}$ such that $0<\xi_{1}<1<\xi_{2}$ and $V^{\prime \prime}\left(\xi_{i}\right)=0, i=1,2$. Furthermore, $V$ is concave on each of the connected components of $\mathbf{R} \backslash\left[\xi_{1}, \xi_{2}\right]$; and $V$ is convex on $\left(\xi_{1}, \xi_{2}\right)$. If $\xi_{2} \geq \lambda^{-1}$. The affinity of $u(x, 1)$, the concave-convex situations as described above, the fact that $u(x, 1)$ is tangent to $V(x)$ at $x=\beta$ and $u(x, 1) \geq v(x, 1)$ at $x=\beta$ and $x=\lambda^{-1}$ imply that $u(x, 1) \geq v(x, 1)$ on $\left(\beta, \lambda^{-1}\right)$. If $\xi_{2}<\lambda^{-1}$. Now $k_{p}\left(\xi_{2}-1\right)^{p-2}=\xi_{2}^{p-2}$ and it follows that $V^{\prime}\left(\xi_{2}\right)=p \xi_{2}^{p-2}<p \lambda^{-(p-2)}$. Consider,

$$
\begin{aligned}
m-p \lambda^{-(p-2)} & =\frac{\lambda}{1-\beta \lambda}\left[\frac{\omega}{\lambda^{p}}-B\right]-p \lambda^{-(p-2)} \\
& =\left\{\frac{\omega-B \lambda^{p}}{1-\beta \lambda}-p \lambda\right\} \lambda^{-(p-1)} \\
& =\frac{p t_{p}\left[p-1-p t_{p}\right]\left[E\left(t_{p}\right)\right]^{2}}{(p-1)\left(1-t_{p}\right)^{2} D\left(t_{p}\right)} \lambda^{-(p-1)}>0
\end{aligned}
$$

from Lemma 5.1(ix), (3.21) and (3.9). Therefore, $m>p \lambda^{-(p-2)}>V^{\prime}\left(\xi_{2}\right)$. Since $u(x, 1) \geq v(x, 1)$ at $x=\beta, x=\xi_{2}$ and $x=\lambda^{-1}$, it follows that $u(x, 1) \geq v(x, 1)$ on $\left(\beta, \lambda^{-1}\right)$. This completes the proof of Case (ii) and hence the proof of Lemma 3.2.

\section{ACKNOWLEDGMENTS}

The author thanks the referee for his comments and suggestions which improved the paper.

\section{REFERENCES}

1. D. L. Burkholder, Martingale transforms, Ann. Math. Statist. 37 (1966), 1494-1504.

2. __ A geometrical characterization of Banach spaces in which martingale difference sequences are unconditional, Ann. Probab. 9 (1981), 997-1011.

3. __ Boundary value problems and sharp inequalities for martingale transforms, Ann. Probab. 12 (1984), 647-702. 
4. $\_$, An elementary proof of an inequality of R. E. A. C. Paley, Bull. London Math. Soc. 17 (1985), 474-478.

5. __ Martingales and Fourier analysis in Banach spaces (C.I.M.E. Lectures, Varenna, Italy, 1985), Lecture Notes in Math., vol. 1206, Springer-Verlag, Berlin and New York, 1986, pp. 61-108.

6. K. P. Choi, Some sharp inequalities for martingale transforms, Ph.D. dissertation, Univ. of Illinois at Urbana-Champaign, 1987.

7. R. C. James, Bases in Banach spaces, Amer. Math. Monthly 89 (1982), 625-640.

8. J. Marcinkiewicz, Quelques théorèmes sur les séries orthogonales, Ann. Soc. Polon. Math. 16 (1937), 84-96.

9. B. Maurey, Système de Haar, Séminaire Maurey-Schwartz (1974-1975), École Polytecnique, Paris.

10. R. E. A. C. Paley, A remarkable series of orthogonal functions. I, Proc. London Math. Soc. 34 (1932), 241-264.

Department of Mathematics, National University of Singapore, Lower Kent Ridge, SINGAPORE 0511 